\title{
La medición del desplazamiento y la difusión de beneficios: Aplicación del método Bowers y Johnson (2003) a la investigación de Cerezo y Díez Ripollés (2010) ${ }^{1}$
}

Manuscrito recibido el 11 enero 2013/Publicado el 17 abril 2013

\section{Carles Soto}

Departamento de Derecho Penal y Criminología, UNED

\begin{abstract}
RESUMEN
La prevención situacional es una forma habitual de prevenir delitos en España pero no se evalúa su eficacia. Una forma de determinar la utilidad de la prevención situacional es calcular el desplazamiento y la difusión de beneficios de la medida preventiva aplicada. Este artículo realiza una evaluación comparativa de la implementación de unas cámaras de vídeo vigilancia instaladas en el centro histórico de Málaga en abril de 2007. Se procede a un reanálisis de los resultados obtenidos por Cerezo y Díez Ripollés en 2010, aplicando el método de cálculo del desplazamiento y difusión de beneficios desarrollados inicialmente por Bowers y Johnson en 2003. El análisis resalta la posibilidad de hacer una evaluación cuantitativa de cualquier programa preventivo que haya obtenido los datos con rigor científico.
\end{abstract}

Palabras clave: evaluación de la prevención situacional, videovigilancia, desplazamiento de delito, difusión de beneficios, cociente ponderado de desplazamiento.

\footnotetext{
${ }^{1}$ La correspondencia debe enviarse a Carles Soto Urpina, Ayuntamiento de Castelldefels, Pl. Iglesia n. ${ }^{\circ}$ 1, 08860 Castelldefels (Spain). carles.soto@ castelldefels.org
} 


\begin{abstract}
Situational crime prevention is a common way of preventing crime in Spain but it has rarely been carefully assessed. One way to know the effectiveness of situational crime prevention is to calculate the displacement and diffusion of benefits of the preventive measure applied. This article reanalyzes the data of the research conducted by Cerezo and Díez Ripollés (2010) on the program of CCTV cameras in Malaga downtown in April 2007. The analysis in based on the model developed by Bowers and Johnson (2003).
\end{abstract}

Key words: effectiveness of situational crime prevention, CCTV cameras, crime displacement, diffusion of benefits, weighted displacement quotient.

\title{
1. Introducción
}

La criminología tiene una amplia diversidad de funciones y todos los paradigmas criminológicos se refieren a la prevención de delito. Pero no basta con reprimir el delito sino que es necesario anticiparse al mismo mediante la prevención. Como afirma López Rey (1975), prevenir es la preparación y la disposición que anticipadamente se hace para evitar que algo acontezca, pero ello nada tiene que ver con curar. La preparación y la disposición presupone un conocimiento lo más claro posible de lo que se quiere prevenir, de los elementos que intervienen, del cuándo o dónde el acontecimiento tendrá probablemente lugar y de la existencia de medios para llevar a cabo las referidas preparación y disposición.

La respuesta tradicional a la prevención del delito ha tenido dos modelos muy semejantes: el clásico y el neoclásico. Actualmente tenemos otro, el situacional, como modelo teórico de prevención. Los modelos tradicionales se basan en la equivalencia entre prevención y disuasión a través del efecto inhibitorio de la pena. El enfoque situacional propugna una intervención especifica dirigida a neutralizar aquellas situaciones de riesgo que ofrece una mayor atractivo al infractor.

El modelo de prevención situacional se inspira en las denominadas teorías del crimen que han supuesto una alternativa a las teorías de la criminalidad que se interesaban en las razones por las que una persona se convierta en delincuente y como rehabilitarlos. La premisa de la teoría de la prevención situacional es que el delito no se distribuye de una manera aleatoria en el espacio y en el tiempo sino que contrariamente 
se produce en lugares y momentos particulares sin perjuicio de tener víctimas $\mathrm{u}$ objetivos con determinadas características.

La prevención situacional es un modelo preventivo que se aplica en diversos entornos de nuestra sociedad aunque no se determina como tal por el desconocimiento de su marco teórico. Básicamente es un sistema de prevención que quiere reducir las oportunidades mediante la modificación del ambiente o las situaciones. La crítica más persistente a la prevención situacional y que ha monopolizado el debate entre partidarios y oponentes de este modelo preventivo es el problema del desplazamiento del delito ya que, según sus opositores, existe una limitada eficacia temporal y transitoria. Los partidarios de la prevención situacional afirman que el efecto del desplazamiento suele ser exagerado y también este enfoque preventivo produce difusión de beneficios.

El desplazamiento no es un simple movimiento de la delincuencia; se trata de una variedad de cambios de conducta como resultado del bloqueo de las oportunidades criminales (Eck, 1993; Barr y Pease, 1990). Cuando hay un bloqueo de las oportunidades delictivas puede haber dos alternativas: si está dentro de la ley se denomina reducción de la delincuencia y si está fuera de la ley, el resultado se conoce como desplazamiento de la delincuencia (Barr y Pease, 1990: 278-279). La mayoría de autores consideran el desplazamiento como el resultado de la aplicación de medidas eficaces contra la delincuencia. El desplazamiento ha sido definido como "un cambio en el comportamiento del delincuente destinado a evitar las medidas preventivas específicas o las condiciones desfavorables del modo habitual de funcionamiento del delincuente" (Gabor, 1990). El Diccionario de la Criminología de Garrido y Gómez (1998) define desplazamiento como el fenómeno por el que el delito reaparece ("se desplaza") después de la introducción de medidas preventivas que incrementan la dificultad para cometer un delito particular o el riesgo de aprehensión del delincuente.

El desplazamiento se ha entendido siempre como una visión negativa de las consecuencias de los esfuerzos de la prevención de la delincuencia pero en algunos casos puede considerarse productor de algunos beneficios (Guerette, 2009a). Las actuales investigaciones sobre el desplazamiento sugieren que el desplazamiento beneficioso o "benigno" puede ocurrir cuando el daño producido por el delito desplazado es un comportamiento problemático menor de lo que existía antes de la intervención (Barr y Pease, 1990; Hesseling, 1995; Eck, 1993; Bowers y Johnson, 2003; 
Guerette y Bowers, 2009; Guerette, 2009b). El desplazamiento se denominará benigno tras la aplicación preventiva de un programa cuando haya un resultado positivo para el conjunto global que compone la delincuencia. El desplazamiento maligno produce más cambios negativos en la delincuencia y trata de cualquier efecto que facilita su reubicación y empeora la situación delictiva.

El estudio del desplazamiento ha encontrado en los últimos años otro beneficio a las medidas de prevención situacional relativo a la difusión de los beneficios. Es la otra cara del desplazamiento, denominado difusión de beneficios, como el efecto positivo general tras la aplicación de medidas preventivas más amplia que la única reducción del delito o delitos concretos originarios del programa. Ha habido varias terminologías utilizadas para describir los efectos de la difusión tras la prevención general de las medidas situacionales: "efecto multiplicador", "efecto halo", "efecto plus" y "efecto aprovechado"2. Clarke y Weisburd definen la difusión de beneficios como la propagación de la influencia beneficiosa de una intervención más allá de los lugares aplicados, de los individuos controlados, los delitos específicos intervenidos o el tiempo de aplicación del programa (Clarke y Weisburd, 1994).

Para realizar la medición del desplazamiento y la difusión de beneficios primero deberemos saber el lugar donde se haya podido mover la delincuencia. Posteriormente se explicarán varios sistemas que cuantifican del desplazamiento y la difusión de beneficios para poder analizar los resultados del programa.

La mayoría de los métodos utilizados para la medición del desplazamiento requiere el uso de al menos tres componentes o áreas (Guerette, 2009a; Bowers y Johnson, 2003):

A.- Área de intervención: es el área donde se introducen las medidas situacionales para provocar cualquier cambio de comportamiento de la delincuencia o actitud frente a un problema. Generalmente se entiende como el área geográfica específica pero también puede ser un sistema, como los autobuses de una ciudad, o un grupo de instalaciones,

\footnotetext{
2 Traducción del inglés de los efectos para describir la prevención general de las medidas situacionales realizada por el autor de este articulo. Los efectos que se refieren a la difusión en el idioma original, inglés, son: "multiplier effect", "halo effect", "bonus effect" y "free-rider". El término "free-rider" ha sido el más difícil de realizar una traducción que se ajustara al contexto y el argot que más se ajusta es el indicado en este artículo, "efecto aprovechado"; otros significados podían ser "efecto vividor" o "efecto gorrón".
} 
como tiendas de una zona, o un periodo de tiempo específico, por ejemplo los sábados por la noche.

B.- Área de desplazamiento/difusión: es la zona que se determina como posible desplazamiento o difusión del delito y/o de la conducta problemática. También se puede entender como un área geográfica que rodea el área de intervención pero puede tener otro sistema (como el traslado de la conducta problemática de un sistema de bus al sistema de metro) o un grupo de instalaciones (tales como tiendas de una zona a domicilios particulares) o de periodos de tiempo (por ejemplo de sábados por la noche a viernes por la noche). En el desplazamiento geográfico, esta área será ubicada alrededor de la zona de intervención y funcionará como amortiguamiento del programa preventivo.

C.- Áreas de control: son las zonas que no aplicaron el programa preventivo y no tuvieron influencia del posible desplazamiento que puede tener la delincuencia. El área de control debe no haber recibido la respuesta de ningún efecto del desplazamiento ni tampoco haber sido influida por otro programa o intervención que pueda alterar los resultados estadísticos. Bowers y Johnson sugieren dos maneras de seleccionar áreas de control ante la posibilidad de desplazamiento y difusión de beneficios: las áreas de control normalmente se ubican alrededor del área de desplazamiento (Figura 1) pero también se utilizan dos o más áreas ubicadas no alrededor del área de intervención pero alejadas prudencialmente (Figura 2). Las áreas de control deben de tener unas características muy parecidas de las áreas de intervención para poder comparar los resultados tras la aplicación de las medidas situacionales (Bowers y Johnson, 2003).

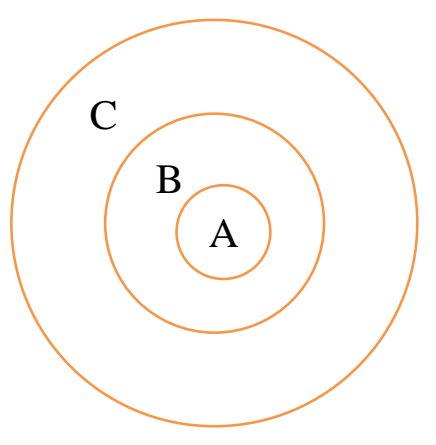

Figura 1

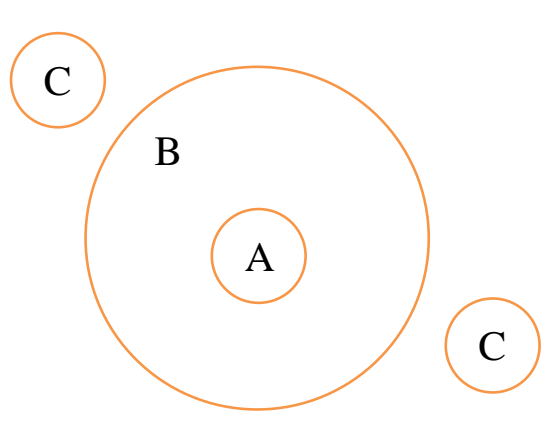

Figura 2 


\section{Marco teórico}

En la medición del desplazamiento y la difusión de beneficios se pueden utilizar varios métodos para poder cuantificar su presencia. Los más usados y consultados en la bibliografía especializada sobre la medición del desplazamiento y difusión son: antes y después, cociente ponderado de desplazamiento, cociente de localización y enfoque componente de Cohort.

\subsection{Antes y después}

Para determinar la presencia de los efectos del desplazamiento y la difusión se pueden tomar medidas antes y después de la implementación de la respuesta en cada área que se identificó en el diseño de la evaluación, explicado en el apartado anterior. Este método permite encontrar cualquier cambio en el comportamiento del problema después de la aplicación de la respuesta con respecto al periodo anterior, facilitando la identificación de los efectos del desplazamiento o la difusión de beneficios.

Se pueden usar diversas medidas para evaluar las mejoras producidas por el proyecto preventivo y a continuación destacaremos las más usuales: (i) El número de delitos por cada 100.000 habitantes; (ii) Los niveles de desorden como el vandalismo o las pintadas; (iii) Analizar la detención de los delincuentes habituales del área analizada y si han sido detenidos en el área de desplazamiento; (iv) Accidentes de tráfico; (v) Índices de daños ocasionados; (vi) Cuantificar los delitos graves.

El método de medición conocido como antes-después, por regla general, es más eficaz cuanto mayor sea el periodo de tiempo empleado para evaluar después de la implementación del programa, siendo habitualmente inferior a dos años. Para muchos proyectos preventivos es suficiente comparar antes y después de los cambios en el área de desplazamiento o zona de difusión para evaluar la eficacia de las medidas situacionales aplicadas. Si hay un aumento del nivel medio de la delincuencia en la zona de desplazamiento/difusión sugiere la producción del efecto analizado en esta investigación, el desplazamiento. Si el aumento es menor que la reducción lograda en el área de intervención, el proyecto aún ha logrado un cierto éxito. Pero si el aumento en el 
área de desplazamiento es mayor que la reducción que el área de intervención, el proyecto no tuvo éxito debido a que el desplazamiento existe. Una disminución en el nivel de delincuencia o problemas de comportamiento en el área de desplazamiento o de difusión sugiere que la difusión se produjo y aumentaron los beneficios del proyecto en el área de intervención.

La Tabla 1 presenta una ilustración de la aplicación práctica del método antesdespués. Por ejemplo, la aplicación de un programa preventivo produjo una reducción de 100 delitos en el área afectada. Un área de desplazamiento/difusión experimentó un aumento de 50 delitos, siendo el desplazamiento. En otra área de desplazamiento/difusión se cuantificó una reducción de 25 delitos, indicando la difusión. La reducción de 100 delitos cuantificados en el área de intervención se reduce a la mitad, 50 delitos, lo cual constituye el desplazamiento ocurrido en un área de desplazamiento/difusión. El efecto de difusión fue de 25 delitos ocurridos en otra área de desplazamiento/difusión y se suma al efecto neto del proyecto, resultando una reducción global de 75 delitos.

Tabla 1. Comparativa de la medición del desplazamiento/difusión utilizando el método antes-después

\begin{tabular}{|l|c|c|c|c|}
\hline & Antes & Después & Diferencia & $\begin{array}{c}\text { Efecto neto } \\
\text { acumulado }\end{array}$ \\
\hline Área de intervención & 200 & 100 & -100 & \\
\hline Área desplazamiento 1 & 100 & 150 & 50 & -50 \\
\hline Área desplazamiento 2 & 75 & 50 & -25 & -75 \\
\hline
\end{tabular}

Muchos estudios empíricos de medición del desplazamiento han utilizado una limitada comparación simple del número de delitos antes y después de la intervención. El resultado estadístico sólo representa los cambios mediante la comparación del área de control con el área de desplazamiento (Bowers y Johnson, 2003). La limitación de este sistema sólo es aplicable a investigaciones a pequeña escala o cuando no es muy 
necesario la precisión de los efectos del desplazamiento y la difusión de beneficios. Este sistema es sencillo y no necesita la participación de analistas avanzados en conocimientos de estadística y evaluación.

\subsection{Cociente de localización}

El ensayo de Barr y Pease en 1990 realiza una medición entre las desigualdades delictivas de unas áreas de estudio. La finalidad de la medición debería ser de ayuda para diseñar políticas preventivas. Los mencionados autores afirman en su estudio que el desplazamiento no se puede calcular por la gran cantidad de factores que implican y lo importante es saber en que tipos de delitos se convierten el desplazamiento y si éstos serán benignos o malignos.

Barr y Pease (1990) proponen una fórmula que no calcula el desplazamiento pero facilita la posibilidad de localización de los actos delictivos en un área, en comparación con el resto de las áreas de la zona. Lo denominan cociente de localización y es un índice que mide la sobrerrepresentación o subrepresentación de algunos fenómenos delictivos en un área de estudio en comparación con una serie de ámbitos. La finalidad del cociente propuesto por Barr y Pease (1990) es localizar las zonas en que ha aumentado o disminuido los delitos después de implementar un programa preventivo. Si el cociente es cero, indica que no hay variabilidad con el resto; si es menos uno, muestra que hay un nivel de delitos estudiados inferior a la media general; si es mayor a cero nos informará que hay un aumento de delitos en la zona. El cociente de localización nos permite evaluar la vulnerabilidad de los individuos en un área y es útil para la evaluación de programas preventivos. Por ejemplo para calcular el cociente de localización de robos en viviendas de un área de estudio se emplearía la siguiente fórmula:

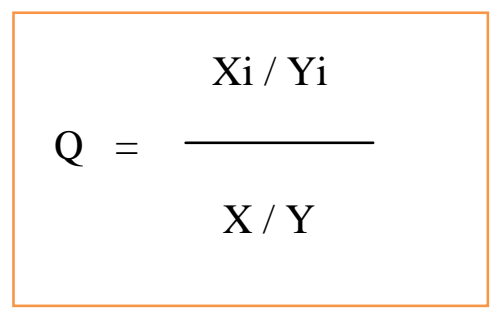


Donde: $\mathrm{Q}=$ Cociente de localización; $\mathrm{Xi}=$ Número robos en vivienda en el área; $\mathrm{X}=$ Número robos en vivienda en todos los ámbitos a considerar; Yi = Número total de viviendas en el área; $\mathrm{Y}$ = Número total de viviendas en todas las área a considerar

\subsection{Enfoque componente de Cohort}

Este modelo ha sido utilizado en demografía para predecir el futuro de la población en un área; por ejemplo, las tasas de fertilidad o mortalidad de una población, las influencias de la inmigración o la emigración. La ecuación utilizada para predecir la población (P1) mediante de este enfoque ha sido:

$$
\mathrm{P} 1=\mathrm{P} 0+(\mathrm{N}-\mathrm{F})+(\mathrm{IN}-\mathrm{EM})
$$

Donde: P0: Población inicial; N: Números de nacimientos ocurridos en el periodo 0 y 1; F: Números de fallecimientos mismo periodo; IN: Número personas que han inmigrado entre 0 y 1; EM: Número de emigrantes mismo periodo; $\mathrm{N}-\mathrm{F}$ : Representa el natural incremento o disminución de la población durante el periodo de estudio; IN EM: Nos indicará la migración existente durante el periodo de estudio y en el área donde se analice.

El enfoque componente Cohort puede servir para saber el impacto de un programa preventivo y estimar cuantitativamente el volumen de desplazamiento que han sufrido los delincuentes. Este enfoque ha sido desarrollado en criminología por Brantingham y Brantingham (2003) aplicando al método inicial las teorías de la criminología ambiental y las investigaciones empíricas sobre los recorridos de los delincuentes. Los mencionados autores afirman que los delitos cometidos en un área son realizados por gente que se mueve por la zona, ya sea como residente o por trabajo o por cualquier actividad general. La ecuación que proponen los autores para entender la reducción de la delincuencia y el desplazamiento tras la intervención de un programa de 
prevención aplicando el modelo del componente Cohort para cuantificar la modificación de la población delictiva (D1) es:

$$
\mathrm{D} 1=\mathrm{D} 0+(\mathrm{N}-\mathrm{V})+(\mathrm{ID}-\mathrm{ED})
$$

Donde: D0: Número de delincuentes que permanecen en activo después de la aplicación del programa preventivo; N: Es la población local que se convierte en delincuente tras la intervención; V: Número de delincuentes que desiste de la actividad delictiva después del programa; ID: Son los delincuente atraídos a la zona tras la intervención preventiva; ED: Son los delincuentes habituales de la zona que se desplazan a otra parte para cometer delitos

Calcular el tamaño de cada población de delincuentes en el tiempo de estudio representa un importante problema según los autores de la ecuación. Las dificultades son metodológicas para calcular las variaciones profesionales de los delincuentes y Brantingham y Brantingham (2003) proponen utilizar los contactos de la policía y los informes de detenciones. Comparto totalmente la dificultad que presenta este enfoque para calcular el desplazamiento por la falta de información sobre la situación de los delincuentes. En muchos países sería muy difícil averiguar el estado delictivo de las personas encausadas en hechos penales. En España sería imposible y podíamos, incluso, estar en situación ilegal en relación a la normativa sobre la protección de datos.

Según los autores de esta ecuación, el desplazamiento es más que la evaluación de un programa preventivo que cuantifica la migración de los delincuentes. El desplazamiento dependerá de la idoneidad de los fines del programa preventivo dirigidos a la reducción del atractivo delictivo de la zona (Brantingham y Brantingham, 2003). Un eficaz programa preventivo producirá la perdida del atractivo delictivo de una zona y los delincuentes que se pueden desplazar (ID) y buscaran otro lugar para ejecutar sus actuaciones delictivas. Además los delincuentes habituales de la zona (ED) se desplazaran a otras zonas donde haya oportunidades más atractivas, desde el punto de vista criminal. La importancia del desistimiento delictivo se observa en la ecuación en lo que denominamos nuevos $(\mathrm{N})$ y viejos $(\mathrm{V})$ : si el programa preventivo es eficaz la 
población del área no se convertirá en delincuente por la falta de oportunidades y los delincuentes de la zona renunciaran a la actividad delictiva. Si estos delincuentes habituales se desplazan (ED) de la zona, entonces el programa preventivo tendría éxito en el ámbito local pero su resultado elevado sería negativo para la evaluación criminológica de la intervención.

Este enfoque es muy general y su interpretación numérica es escasa por las dificultades explicadas anteriormente y por el trabajo de campo que se necesitaría para su aplicación. Los cálculos de cambios personales de los delincuentes son muy variables y depende de muchos factores sociales, alterándose en breves espacios de tiempo, causando muchos falsos positivos o negativos.

\subsection{Cociente ponderado de desplazamiento (CPD)}

Este método de medición del desplazamiento y la difusión de beneficios fue sugerido por Kate J. Bowers y Shame D. Johnson en 2003. Estos autores desarrollaron una serie de formulas para cuantificar los efectos del desplazamiento y la difusión en relación con las respuestas del programa preventivo. El CPD se basa en la división de las áreas explicadas anteriormente y analiza los resultados de las tres zonas para calcular el desplazamiento o la difusión de beneficios. La razón de este sistema de medición se fundamenta en que durante el tiempo de aplicación del programa el área de desplazamiento recibe una determinada proporción de delitos del área de intervención; su cálculo se pondera con los delitos que se cometen en la zona de control. Si existe desplazamiento aumentarán los delitos en el área de desplazamiento y disminuirán en el área de intervención, pero teniendo en cuenta que el área de control no se percibe un cambio proporcional de delitos.

Para el cálculo del desplazamiento o difusión en la fórmula propuesta por los mencionados autores sugieren cuatro cálculos:

Primero, averiguar los cambios antes y después de la intervención, denomina efecto bruto (EB), siendo la resta de los delitos que hubo antes y después en la zona de intervención, área A: 


$$
\mathrm{EB}=\mathrm{Aa}-\mathrm{Ad}
$$

Si el resultado es positivo nos indicará un descenso del problema; el resultado cero señalará que no hay cambio y un número negativo nos mostrará que la situación empeoró.

Segundo, denominado efecto neto (EN), nos mostrará si los cambios producidos en el área de intervención son consecuencia de la aplicación del programa preventivo, comparándolo con los cambios ocurridos en el área de control, área C:

$$
\mathrm{EN}=\frac{\mathrm{Aa}}{\mathrm{Ca}}-\frac{\mathrm{Ad}}{\mathrm{Cd}}
$$

Si el resultado es cero indicará que posiblemente el programa aplicado no haya sido eficaz y si es negativo nos mostrará que las cosas han empeorado con la intervención. Ambos resultados puntualizarán que el desplazamiento y la difusión son irrelevantes así que no deberíamos continuar analizando los resultados del programa. En cambio si el resultado es positivo, habrá razones para entender que la intervención pudo causar la mejoría del área de intervención. Por tanto podemos seguir analizando la existencia del desplazamiento o la difusión con el cálculo del CPD.

Tercero, se incorporaran las infracciones ocurridas en el área de desplazamiento/difusión, área $\mathrm{B}$, antes y después de la implantación de la medida preventiva. Y se buscará la medición del desplazamiento o la difusión que ha producido el programa preventivo en el área de desplazamiento, mediante el cálculo del Cociente ponderado de desplazamiento (CPD):

$$
\mathrm{CPD}=\frac{\mathrm{Bd} / \mathrm{Cd}-\mathrm{Ba} / \mathrm{Ca}}{\mathrm{Ad} / \mathrm{Cd}-\mathrm{Aa} / \mathrm{Ca}}
$$

La parte superior (numerador) muestra el número aproximado de difusión o desplazamiento; si es negativo indicará difusión y si es positivo indica desplazamiento. 
La parte inferior (denominador) mide la eficacia de la respuesta en relación con el control; un programa eficaz producirá un número negativo. Si el denominador es positivo significará la dificultad de relacionar cualquier cambio en el área de desplazamiento con el programa preventivo, siendo inadecuado teóricamente utilizar CPD para buscar el desplazamiento/difusión de beneficios.

Si el resultado de CPD es igual a cero no habrá volumen de desplazamiento o difusión. Si el CPD es positivo, hay difusión (teniendo en cuenta que la división de dos números (dividendo y divisor) negativos dará un resultado con valor positivo); y si es superior a 1 nos mostrará que el efecto de la difusión es mayor que el de la intervención. Si el resultado CPD es negativo, habrá desplazamiento; entre 0 y -1, el desplazamiento contrarresta algunos efectos de la intervención pero no todos. Si el CPD es menor a -1 indicará que la respuesta empeoró la situación.

Tabla 2. Interpretación del Cociente ponderado de desplazamiento

\begin{tabular}{|c|c|c|}
\hline Valor $\boldsymbol{C P D}$ & Efecto & Interpretación \\
\hline $\boldsymbol{C P D}>\mathbf{1}$ & Difusión mayor que los efectos directos & ++ \\
\hline $\boldsymbol{C P D}$ cerca $\mathbf{1}$ & Difusión más o menos igual a los efectos directos & + \\
\hline $\mathbf{1} \boldsymbol{C P D}>\mathbf{0}$ & Difusión pero menos que los efectos directos & + \\
\hline $\boldsymbol{C P D}=\mathbf{0}$ & No desplazamiento o difusión & + \\
\hline $\mathbf{0}>\boldsymbol{C P D}>-\mathbf{1}$ & Desplazamiento pero menos que los efectos directos & + \\
\hline $\boldsymbol{C P D}$ cerca $-\mathbf{1}$ & Desplazamiento casi igual efectos directos \\
\hline $\boldsymbol{C P D}<-\mathbf{1}$ & Desplazamiento mayor que los efectos directos & - \\
\hline
\end{tabular}

Fuente: Bowers y Johnson, 2003 
El CPD puede dividirse en diferentes medidas para calcular el éxito de la intervención y el desplazamiento/difusión que se haya producido:

- Medición del éxito: será el denominador del CPD

$$
\mathrm{Ad} / \mathrm{Cd}-\mathrm{Aa} / \mathrm{Ca}
$$

Si esta medición es negativa demostrará que el programa preventivo fue exitoso con la reducción del delito en comparación con el área de control.

- Medición del desplazamiento: interpretado por el numerador del CPD

$$
\mathrm{Bd} / \mathrm{Cd}-\mathrm{Ba} / \mathrm{Ca}
$$

Si el resultado es positivo nos indicará la existencia de desplazamiento; sin embargo, si es negativo sugerirá la posibilidad de difusión de beneficios, ya que el área de desplazamiento ha tenido, después de la implementación del programa preventivo, menos índice delictivo analizado, en comparación con el área de control.

Cuarto, el impacto global del proyecto se puede determinar, según el ensayo de Bowers y Johnson, mediante el Efecto neto total (ENT) del programa preventivo:

$$
\mathrm{ENT}=[\mathrm{Aa}(\mathrm{Cd} / \mathrm{Ca})-\mathrm{Ad}]+[\mathrm{Ba}(\mathrm{Cd} / \mathrm{Ca})-\mathrm{Bd}]
$$

La primera parte de la formula indica la eficacia del programa preventivo en el área de intervención; cuanto más eficaz hayan sido las medidas preventivas aplicadas, más grande será este término. La segunda parte muestra el nivel de difusión o desplazamiento; positivo indicará difusión y negativo mostrará desplazamiento. Mientras más positivo sea el ENT más eficaz habrá sido el programa de prevención situacional. Según los especialistas de la aplicación de estas fórmulas de cálculo del desplazamiento y la difusión de beneficios, el número obtenido con ENT significaría la cantidad de delitos prevenidos tras la implementación del proyecto de prevención situacional en la zona (Guerette, 2009a). 
La medición del desplazamiento y la difusión de beneficios presentan varios problemas, según el ensayo de Bowers y Johnson de 2003: (i) Saber exactamente cómo ha cambiado el comportamiento de los delincuentes; (ii) Cuantificar en términos absolutos el desplazamiento producido, así como la cantidad de delitos que se han evitado mediante la disuasión de las medidas preventivas implementadas; (iii) Incluso con la existencia de desplazamiento, es muy difícil saber los beneficios económicos obtenidos por los delincuentes para saber si ha habido un aumento o disminución de ganancias procedentes de los delitos.

El CPD debe ser interpretado con cautela y entender que fue desarrollado para responder a la existencia del desplazamiento o difusión de beneficios, en lugar de cuantificar en términos absolutos ambos efectos. Sin embargo es posible interpretar el cociente con un poco más de detalle como ilustra la Tabla 2.

Muchos estudios empíricos utilizan para la medición del desplazamiento y la difusión el método de análisis de series de tiempo, que se limitan a comparar el número de hechos delictivos antes y después de una intervención. El método de medición propuesto por Bowers y Johnson de 2003 y utilizado por varias investigaciones criminológicas presenta una serie de ventajas:

(i) Prevalece el éxito del plan preventivo mediante el cociente ponderado frente al cálculo cuántico del desplazamiento del delito.

(ii) Minimiza el problema de calcular los delitos desplazados ya que es una medida relativa que no hace suposiciones acerca de números absolutos de delitos.

(iii) Examina tasas de delincuencia y no volúmenes reales de delitos; por tanto los datos están estandarizados y permite comparaciones.

(iv) El CPD es una medida totalmente integrada que representa las tendencias delictivas en todas las zonas, incluso las áreas de control.

(v) No sólo se basa en la medición del desplazamiento/difusión en la zona de amortiguamiento, área $\mathrm{B}$, sino que mide la delincuencia en las zonas de control, área $\mathrm{C}$, que pueden variar por diversas razones y no son reflejadas en la zona de amortiguamiento. 


\section{Resultados}

Para analizar el desplazamiento y la difusión de beneficios vamos a utilizar los datos empíricos del trabajo realizado por Cerezo y Díez Ripollés (2010) sobre la eficacia de la videovigilancia en la reducción de la delincuencia. Esta investigación no está dirigida exclusivamente hacia el análisis del desplazamiento pero han realizado una excelente recopilación de datos temporales para su correcto análisis científico. Por la validez metodológica de los datos y su acotamiento temporal he creído oportuno utilizar la supracitada investigación para demostrar la viabilidad del método sugerido por Bowers y Johnson en 2003 en la medición del desplazamiento y la difusión de beneficios.

3.1. Estudio de Cerezo y Díez Ripollés (2010) sobre la efectividad de la videovigilancia en la reducción de la delincuencia

El estudio que vamos a comentar sobre la eficacia de la videovigilancia en la reducción de la delincuencia es una de las pocas investigaciones españolas donde se analiza con cierta entidad el desplazamiento espacial. Cerezo y Díez Ripollés son dos conocidos investigadores de la criminología española y pertenecen al Instituto Andaluz Interuniversitario de Criminología.

La investigación se presentó en el VII Congreso Español de Criminología realizado en Elche en junio de 2010. El objetivo de los investigadores era evaluar la eficacia de las 41 cámaras de videovigilancia instaladas en el centro histórico de Málaga en abril de 2007. Los profesores realizaron varias hipótesis en la investigación pero en este trabajo sólo desarrollaremos la segunda: el desplazamiento del delito tras la instalación de unas cámaras de videovigilancia.

La metodología de la investigación fue cuantitativa y cualitativa: tasas de delincuencia procedente de registros oficiales, datos de encuestas de victimización y entrevistas en profundidad con comerciantes y operadores del sistema de videovigilancia.

Cerezo y Díez Ripollés utilizan para la investigación que estamos estudiando el método de análisis antes y después que tan necesario es en las ciencias sociales. Aunque parece sencillo, muchas investigaciones criminológicas españolas no tienen más 
reconocimiento porque no se acotan en el tiempo y los resultados no pueden ser comparados, llegando a conclusiones de lógica sin amparo objetivo. La investigación acota los datos en abril de 2007 y realiza una investigación de los hechos antes de la mencionada fecha, de abril 2006 hasta marzo de 2007, y vuelve a analizar datos, encuestas y entrevistas desde abril 2007 hasta marzo 2008.

La investigación analizó 6.245 delitos y distribuyeron geográficamente las infracciones en dos áreas: área de tratamiento, área de control y cuatro zonas: 10 calles con cámaras, 10 calles cerca de cámaras, 10 calles con características similares a las calles con cámaras y 10 calles con características similares a las calles cerca de cámaras.

Los datos policiales obtenidos en la ciudad de Málaga eran de 3.048 infracciones ocurridas en el primer año de estudio y 3.197 durante el segundo año. La distribución temporal y geográfica de las infracciones en las zonas de estudio se expone detalladamente en la siguiente Tabla 3.

Tabla 3. Número total de infracciones por zonas y periodo temporal de estudio (antes y después de la instalación de las cámaras)

\begin{tabular}{|l|c|c|c|}
\hline \multicolumn{1}{|c|}{ ZONAS } & $\begin{array}{c}\text { AÑO 1 } \\
\text { (antes) }\end{array}$ & $\begin{array}{c}\text { AÑO 2 } \\
\text { (después) }\end{array}$ & TOTAL \\
\hline $\begin{array}{l}\text { 10 calles con cámaras } \\
\text { (área de tratamiento) }\end{array}$ & 982 & 963 & $\begin{array}{c}-19 \\
\mathbf{- 1 , 9 \% )}\end{array}$ \\
\hline $\begin{array}{l}\text { 10 calles cerca de cámaras } \\
\text { (área de tratamiento) }\end{array}$ & 755 & 852 & $\begin{array}{c}+\mathbf{1 1 0} \\
\mathbf{+ + 1 4 , 6 \% )}\end{array}$ \\
\hline $\begin{array}{l}\text { 10 calles con características similares a } \\
\text { las calles con cámaras } \\
\text { (área de control) }\end{array}$ & 560 & 622 & $\begin{array}{c}+\mathbf{6 2} \\
\mathbf{+ 1 1 , 1 \% )}\end{array}$ \\
\hline $\begin{array}{l}\text { 10 calles con características similares a } \\
\text { las calles cerca de cámaras } \\
\text { (área de control) }\end{array}$ & $\mathbf{7 5 1}$ & $\mathbf{7 4 0}$ & $\begin{array}{c}\mathbf{- 1 1} \\
\mathbf{- 1 , 4 \% )}\end{array}$ \\
\hline
\end{tabular}


Si analizamos los datos de la tabla, los resultados constatan un aumento de las infracciones después de la instalación de las cámaras de videovigilancia hacia las 10 calles cerca de las cámaras, $+14,6 \%$, y hacia las 10 calles con características similares a las calles con cámaras, $+11,1 \%$. Se observa una disminución de $-1,9 \%$ de los delitos en las 10 calles con cámaras. También parece que la estadística general sobre los delitos en la ciudad de Málaga es de disminución entre las dos épocas de estudio, ya que las 10 calles con características similares a las calles cerca de las cámaras ha habido una disminución de la delincuencia del -1,4\%.

La tasa de victimización general comparando las dos periodos de estudio se ha visto reducida. Se ha producido un ligero descenso del índice de victimización entre los años 2006 y 2008: de una tasa de 20,2 \% de malagueños que aseguran haber sufrido un delito durante los 12 meses anteriores a marzo 2006 se reduce a una tasa de victimización de 19,4\% que afirmaron lo mismo en la segunda encuesta realizada en marzo de 2008 (Cerezo y Díez Ripollés, 2011).

En la percepción de la delincuencia y el miedo al delito no se aprecia un cambio significativo entre los ciudadanos entrevistados antes y después de la instalación de las cámaras. En cambio, la sensación de los comerciantes con establecimientos en las calles del centro histórico con cámaras es de mayor seguridad.

Las conclusiones que desprenden los investigadores andaluces sobre el desplazamiento es que tras la implementación de una medida preventiva situacional, como son las cámaras de videovigilancia, algunos delitos se desplazan hacia las calles colindantes de la zona de intervención o hacia las calles con características similares a las calles con cámaras.

El estudio de Cerezo y Díez Ripollés no tenía como objetivo exclusivo estudiar el desplazamiento sino era una investigación sobre la efectividad de la videovigilancia en la reducción de la delincuencia. Las primeras investigaciones empíricas sobre el desplazamiento realizadas por John Eck en 1993 o René Hesseling en 1994 tuvieron mucha crítica por no ser exclusivos sobre el análisis del desplazamiento. Estos dos primeros estudios analizaron el desplazamiento como un aspecto más de la investigación y el objetivo principal era evaluar la eficacia de una medida de prevención situacional; además no realizaron ninguna aportación sobre la difusión de beneficios. 
Los autores Cerezo y Díez Ripollés tampoco incorporan ninguna explicación sobre la difusión de beneficio después de la implementación de la medida de prevención situacional en el centro de Málaga. Aunque concluyen en su estudio que la existencia de las cámaras en algunas calles del centro histórico ofrece a los comerciantes una mayor tranquilidad. Cerezo y Díez Ripollés también comprobaron que había un ligero decremento del índice de victimización entre los años 2006 y 2008, siendo otro ejemplo de difusión de beneficios. Añadir que esta influencia positiva es baja, ya que 20,2\% los ciudadanos entrevistados en el año 2006 aseguraron haber sufrido un delito y en el año 2008 los afirmaron un 19,4\%. Y tenemos que tener en cuenta que las cámaras instaladas en el centro histórico de Málaga en abril de 2007 apenas habían reducido la delincuencia en las calles donde habían sido ubicadas, siendo de $-1,9 \%$ según el estudio publicado en 2010.

\subsection{La medición del desplazamiento y la difusión de beneficios tras la} instalación de cámaras de videovigilancia en el centro de Málaga en abril 2007 utilizando el método sugerido por Bowers y Johnson en 2003

Se procede continuación incorporar el método de medición del desplazamiento y la difusión de beneficios sugerido por Bowers y Johnson en 2003 y poder cuantificar su efecto con los datos obtenidos en Málaga tras la implantación de una medida de prevención situacional, como son 42 cámaras de videovigilancia. Hay que destacar que el método propuesto por Bowers y Johnson está diseñado principalmente para evaluar la eficacia de un programa preventivo mediante el análisis del desplazamiento y la difusión de beneficios El estudio de Cerezo y Díez Ripollés tiene como objetivos evaluar la eficacia de la videovigilancia en la reducción de la delincuencia, el análisis del desplazamiento y el estudio del sentimiento de seguridad.

Primero deberíamos utilizar la distribución de las tres áreas de medición (intervención, desplazamiento/difusión, control) explicadas en este trabajo y utilizada en diversas investigaciones específicas sobre el desplazamiento y la difusión de beneficios. Esta distribución aplicada al estudio realizado en Málaga sería el siguiente:

- Área de intervención: 10 calles con cámaras 
- Área de desplazamiento/difusión: 10 calles cerca de cámaras

- Área de control: 10 calles con características similares a las calles cerca de cámaras

Las dos primeras áreas son evidentes su similitud a las clasificadas mediante el método sugerido por Bowers y Johnson. El área de control ha sido seleccionada por las 10 calles con características similares a las calles cerca de las cámaras por que no han tenido ninguna influencia de la implementación de las cámaras de videovigilancia. Bowers y Johnson siempre han afirmando que el área de control no puede tener aplicación del programa preventivo ni influencia del posible desplazamiento que haya sufrido la delincuencia. Además, Cerezo y Díez Ripollés afirman en sus conclusiones que el área de control que presenta características similares a las calles con cámaras se ha comprobado el desplazamiento. En resumen, el área de control seleccionada no ha estado influenciada por la implantación de las 41 cámaras de videovigilancia del centro de Málaga.

Después de las aclaraciones realizadas sobre la distribución de las áreas de medición, los resultados cuantitativos obtenidos para la medición del desplazamiento y la difusión de beneficios se exponen en la Tabla 4. 
Tabla 4. Número total de infracciones por zonas y periodo temporal de estudio (Antes y después de la instalación de las cámaras)

\begin{tabular}{|l|c|c|c|}
\hline \multicolumn{1}{|c|}{ ZONAS } & $\begin{array}{c}\text { AÑO 1 } \\
\text { (antes) }\end{array}$ & $\begin{array}{c}\text { AÑO 2 } \\
\text { (después) }\end{array}$ & TOTAL \\
\hline $\begin{array}{l}\text { A.- Área de intervención } \\
\text { (10 calles con cámaras) }\end{array}$ & 982 & 963 & $\begin{array}{c}-19 \\
\mathbf{- 1 , 9 \% )}\end{array}$ \\
\hline $\begin{array}{l}\text { B.-Área de desplazamiento } \\
(10 \text { calles cerca de cámaras) }\end{array}$ & 755 & 852 & $\begin{array}{c}+110 \\
\mathbf{+ 1 4 , 6 \% )}\end{array}$ \\
\hline $\begin{array}{l}\text { C. } \text { - Área de control } \\
\text { (10 calles con características } \\
\text { similares a las calles cerca de } \\
\text { cámaras) }\end{array}$ & $\mathbf{7 5 1}$ & $\mathbf{7 4 0}$ & $\begin{array}{c}-\mathbf{1 1} \\
\mathbf{( - 1 , 4 \% )}\end{array}$ \\
\hline
\end{tabular}

Fuente: Elaboración propia

Aplicando el método de medición del desplazamiento y la difusión de beneficios de Bowers y Johnson (2003) a los datos de la Tabla 4 podemos destacar los siguientes resultados:

- $\mathrm{EB}=19 \mathrm{El}$ efecto bruto nos indica los cambios antes y después de la intervención; si el resultado es positivo nos indica un descenso del problema en el área de intervención.

$$
\mathrm{EB}=\mathrm{Aa}-\mathrm{Ad}=982-963=19
$$

- $\mathrm{EN}=0,0062 \mathrm{El}$ efecto neto nos mostrará los cambios producidos en el área de intervención como consecuencia de la aplicación del programa preventivo. Si el resultado es positivo entenderemos que la intervención pudo causar la mejoría en el área de intervención.

$$
\mathrm{EN}=\mathrm{Aa} / \mathrm{Ca}-\mathrm{Ad} / \mathrm{Cd}=982 / 751-963 / 740=1,3075-1,3013=0,0062
$$


- $\mathrm{CPD}=-23,5438$, el cociente ponderado de desplazamiento busca la medición del desplazamiento o la difusión de los beneficios. Si el resultado es negativo nos indicara que hay desplazamiento y si es mayor a -1 , como el actual caso, nos mostrará que el mencionado desplazamiento ha sido mayor que los efectos directos, teniendo una interpretación negativa de la eficacia de la instalación de las cámaras de videovigilancia.

$\mathrm{CPD}=\frac{\mathrm{Bd} / \mathrm{Cd}-\mathrm{Ba} / \mathrm{Ca}}{\mathrm{Ad} / \mathrm{Cd}-\mathrm{Aa} / \mathrm{Ca}}=\frac{852 / 740-755 / 751}{963 / 740-982 / 751}=\frac{1,1513-1,0053}{1,3013-1,3075}=\frac{0,146}{-0,0062}=-23,5438$

El CPD puede dividirse en diferentes medidas para calcular el éxito de la instalación de las cámaras de videovigilancia y el desplazamiento / difusión que se haya producido:

- Medición del éxito: será el denominador del CPD, siendo igual a -0,0062. Si esta medición es negativa demostrará que la instalación de las cámaras de videovigilancia fue exitosa con la reducción del delito en comparación con el área de control.

- Medición del desplazamiento: interpretado por el numerador del CPD, siendo igual a 0,146. Si el resultado es positivo nos indicará la existencia de desplazamiento en el estudio de los profesores Cerezo y Díez Ripollés.

- $\mathrm{ENT}=-103,5339$, el efecto neto total determina el impacto global del proyecto. Según los investigadores practicantes de esta forma de medición del desplazamiento y la difusión de beneficios (Guerette, 2009a), el número obtenido significaría la cantidad de delitos prevenidos tras la instalación de las cámaras de videovigilancia en el centro de Málaga en abril de 2007. Según el resultado negativo obtenido se entenderá que no se ha prevenido ningún delito. 


$$
\begin{aligned}
\mathrm{ENT}= & {[\mathrm{Aa}(\mathrm{Cd} / \mathrm{Ca})-\mathrm{Ad}]+[\mathrm{Ba}(\mathrm{Cd} / \mathrm{Ca})-\mathrm{Bd}]=[982(740 / 751)-963]+} \\
& {[755(740 / 751)-852]=4,5646+(-108,0985)=-103,5339 }
\end{aligned}
$$

El ENT consta de dos partes que miden la eficacia de la instalación de las cámaras de videovigilancia en el área de intervención y el nivel de difusión o desplazamiento de la siguiente manera:

- Medición de la eficacia de la medida preventiva en el área de intervención: interpretado por la primera parte de la fórmula y con un resultado de 4,5676. Cuanto más eficaz haya sido la implementación de las cámaras de videovigilancia en el centro de Málaga más grande será este término.

- Nivel de difusión o desplazamiento: lo determinará la segunda parte de la fórmula y el resultado ha sido - 108,0985. Cuando esta segunda parte de la fórmula es negativa nos indicará que ha habido desplazamiento por la instalación de las cámaras de videovigilancia.

La evaluación sobre la implementación de unas cámaras de videovigilancia instaladas en el centro histórico de Málaga en abril de 2007 aplicando el método sugerido por Bowers y Johnson en 2003 nos informa que ha habido un desplazamiento. Este cociente nos indica que hubo reducción del delito después de la implementación de la medida preventiva en una cantidad de - 0,0062, siendo mínima esta mejoría. También hubo desplazamiento tras la instalación de las cámaras de videovigilancia en 0,146. Estas cuantificaciones serian interesantes de utilizar para poder realizar comparativas con otras investigaciones sobre programas o medidas de prevención implementadas y evaluar con el mismo procedimiento la eficacia preventiva. El estudio que he realizado concluye que la instalación de las cámaras de videovigilancia en Málaga durante el año 2007 ha producido desplazamiento de la delincuencia con un cociente ponderado de 23,54, utilizando el método Bowers y Johnson (2003). 


\section{Conclusiones}

Los resultados obtenidos de la investigación de los profesores Cerezo y Díez Ripollés (2010) sobre la eficacia de las cámaras de videovigilancia instaladas en el centro de Málaga en abril de 2007 y el presente estudio aplicando el método de medición del desplazamiento y la difusión de beneficios sugerido por Bowers y Johnson en 2003 concuerdan.

Con el método sugerido por Bowers y Johnson en 2003 podemos evaluar la eficacia de programas preventivos en una misma forma por que los datos están estandarizados y permite comparaciones. El método sugerido por Bowers y Johnson minimiza los problemas de cuantitativos de los delitos desplazados ya que es una medida relativa que no hace suposiciones acerca de números absolutos de delitos. El objetivo no es cuantificar el desplazamiento del delito sino evaluar el éxito del plan preventivo mediante el CPD.

La instalación de las mencionadas cámaras en el área de intervención comportó un descenso del problema $(\mathrm{EB}=19)$ y una mejoría de la situación $(\mathrm{EN}=0,0062)$; hubo una pequeña mejoría en la reducción del delito en el área de intervención en relación al área de control, como queda evidente en el estudio de Cerezo y Díez Ripollés. También se confirma, como afirmaban los profesores Cerezo y Díez Ripollés, la mínima eficacia de la instalación de las cámaras de videovigilancia en el centro de Málaga en el año 2007 expresado en la primera parte del ENT, siendo 4,5676 una cantidad muy escasa.

Los investigadores Cerezo y Díez Ripollés confirman textualmente "un posible efecto de desplazamiento del delito". La utilización de la metodología propuesta por Bowers y Johnson nos confirma que el cociente ponderado de desplazamiento en 23,5438, siendo mayor el desplazamiento que los efectos de la difusión de beneficios obtenidos por la instalación de las cámaras. El cálculo ponderado del desplazamiento que se realiza en este estudio se tiene en cuenta la difusión de beneficios que produce la instalación de las cámaras de videovigilancia y al ser negativo nos indicará que ha habido más desplazamiento que difusión de beneficios.

Actualmente en España no evaluamos nuestros sistemas de control con rigor científico e incluso muchos sectores de la Administración Pública encargados de los sistemas de control, en general, y los sistemas de seguridad, en particular, no quieren 
que se puedan evaluar sus acciones y menos sus decisiones, como puede ser la instalación de unas cámaras de videovigilancia. Málaga y los profesores Cerezo y Díez Ripollés han sido una excepción y por tal razón se ha podido aplicar las fórmulas sugeridas por Bowers y Johnson en 2003 sobre el desplazamiento y la difusión de beneficios.

Me gustaría destacar del presente análisis que he realizado sobre la instalación de las cámaras de videovigilancia no el resultado sino más bien la metodología y la posibilidad que con datos recogidos científicamente con acotamiento temporal y territorial se puede realizar una investigación concluyente sobre el desplazamiento y la difusión de beneficios. Con el método sugerido por Bowers y Johnson podemos hacer comparaciones por que se evalúa programas preventivos con el mismo procedimiento.

\section{Agradecimientos}

Quisiera agradecer el asesoramiento y las sugerencias del Dr. Carlos Vázquez González del Departamento de Derecho Penal y Criminología de la UNED que han mejorado sustancialmente el presente artículo. También quisiera agradecer los comentarios y recomendaciones realizados por el editor de la revista y los revisores anónimos de la REIC.

\section{Referencias}

Barr, R. y Pease, K. (1990). Crime placement, displacement and deflection. En M. Tonry \& N. Morris (Eds.), Crime and justice: A review of research, vol. 12 (pp. 277-318). Chicago: University of Chicago Press.

Bowers, K. J. y Johnson, S. D. (2003). Measuring the geographical displacement and diffusion of benefit effects of crime prevention activity. Journal of Quantitative Criminology, 19(3), 275-301.

Brantingham, P. L. y Brantingham, P. J. (2003). Crime prevention and the problem of crime displacement: Estimating quantum of displacement using a cohort component approach. En H. Kury, \& J. Obergfell-Fuchs (Eds.), Crime prevention: New approaches (pp. 365-369). Mainz: Weisser Ring.

Cerezo Domínguez, A. I. y Díez Ripollés, J. L. (2010). La videovigilancia en las zonas públicas: su eficacia en la reducción de la delincuencia. Boletín Criminológico, $121,1-4$. 
Cerezo Domínguez, A. I. y Díez Ripollés, J. L. (2011). Videocámaras y prevención de la delincuencia en lugares públicos: análisis jurídico y criminológico. Valencia: Tirant lo Blanch.

Clarke, R. V. Y Weisburd, D. (1994). Diffusion of crime control benefits: Observations on the reverse of displacement. En R. V. Clarke (Ed.), Crime prevention studies, vol. 2 (pp. 165-182). Monsey, NY: Criminal Justice Press.

Eck, J. E. (1993). The threat of crime displacement. Criminal Justice Abstract, 253, 527-46.

Gabor, T. (1990). Crime displacement and situational prevention: Toward the development of some principles. Canadian Journal of Criminology, 32, 41-74.

Garrido Genovés, V. y Gómez Piñana, A.M. (1998). Diccionario de Criminología. Valencia: Tirant lo Blanc.

Guerette, R. T. (2009a). Analyzing crime displacement and diffusion. ProblemOriented Guides for Police Problem-Solving Tools Series, 10. Obtenido de http://www.popcenter.org/tools/pdfs/displacement.pdf.

Guerette, R. T. (2009b). The pull, push, and expansion of situational crime prevention evaluation: An appraisal of thirty-seven years of research. Crime Prevention Studies, 24, 29-58.

Guerette, R. T., \& Bowers, K. J. (2009). Assessing the extent of crime displacement and diffusion of benefits: A review of situational crime prevention evaluations. Criminology, 47(4), 1331-1368.

Hesseling, R. B. P. (1994). Displacement: A review of the empirical literature. En R. V. Clarke (Ed.), Crime prevention studies, vol. 3, pp. 197-230. Monseny, NY: Criminal Justice Press.

López Rey, M. (1975). Teoría, delincuencia juvenil, prevención, predicción y tratamiento. Madrid: Aguilar.

Carles Soto es el responsable del Observatorio de la Delincuencia del ayuntamiento de Castelldefels y sus campo de interés son la prevención de la delincuencia y la teoría criminológica. Es Licenciado en Criminología por la Universidad de Barcelona y Diplomado en Estudios Avanzados en el doctorado de Derecho Penal y Criminología de la UNED. Actualmente está realizando la tesis doctoral sobre la prevención situacional, concretamente en el análisis del desplazamiento y la difusión de beneficios. Es vicepresidente de la Asociación Catalana de Criminólogos. 